\title{
Studies on Genetic Variability, Hertability and Genetic Advance in Turmeric (Curcuma longa L.)
}

\author{
Poonam*, I. B. Maurya, Manoj Sharma, A. Kavita, Bhim Singh, \\ Bhuri Singh, Priyanka Kumawat and Anita Verma
}

College of Horticulture \& Forestry, Jhalarapatan, Jhalawar- 326 023, Rajasthan, India

*Corresponding author

\section{A B S T R A C T}

\section{Keywords}

Genetic Variability

Hertability

Genetic Advance

Turmeric

Article Info

Accepted:

24 June 2018

Available Online:

10 July 2018
The present investigation was carried out to estimate the genetic variability, heritability and genetic advance analysis among 25 genotypes of turmeric for rhizomes yield and its contributing characters. These genotypes were planned in Randomized Block Design with three replications during June, 2017 to March, 2018 at Protected Cultivation Unit of the College of Horticulture and Forestry, Jhalawar, Rajasthan. The analysis of variance indicated presence of considerable variability for all the 21 characters. High genotypic coefficient of variance (GCV) and phenotypic coefficient of variation (PCV) were recorded for per cent fresh weight of secondary rhizomes per plant followed by dry weight of rhizomes per plant, number of tillers per plant and length of mother rhizomes per plant. Therefore, these characters can be improved through selection programme.

\section{Introduction}

Turmeric (Curcuma longa L.) a perennial herbaceous plant belonging to the family Zingiberaceae under the natural orders Scitaminae. Its native of South East Asia and chromosome number is $2 \mathrm{n}=32$. The karyomorphological studies concluded that the species seems to be allotetraploid with basic chromosome number of $X=8$ (Sato, 1960). It is cultivated for its underground stem called as rhizomes, which are used for medicinal and culinary purpose and also as a cosmetic and a natural dye. It is grown in an area of 233 thousand hectares with an average of annual production of 1190 thousand tones.

It is a principal ingredient in curry powder. Turmeric oleoresin is used in brine pickles and to some extent in non-alcoholic beverages, gelatins, butter and cheese etc. It is used in the preparation of medicinal oils, ointments and poultice. It is also used in stomachic, carminative, tonic, blood purifier and an antiseptic. The aqueous extracts have biopesticidal properties (Prajapati et al., 2014). 
Turmeric of commerce is valued for its deep yellow colour and pungent aromatic flavor due to the presence of colouring matter 'curcumin' and a volatile oil 'terminol'. Curcumin content present in turmeric range between 3.5 to $9 \%$.

Quality of turmeric depends on its size, colour, dry matter content and number of rhizomes per plant etc. Turmeric is cultivated in many parts of countries and has wide range of variability among the different quantitative and qualitative characters. The critical assessment of variability is prerequisite for any efficient breeding programme and provides opportunity to identify the superior genotypes with desirable yield, related traits and quality characters. The turmeric is mainly propagated asexually through rhizomes, some varieties or genotypes produce flowers but these flowers do not set seed. Not much work is carried out on crop improvement of turmeric in India. There is no planned multiplication programmes for planting material. Hence, present investigation was under taken to study the genetic variability present in different genotypes of turmeric.

\section{Materials and Methods}

The planting material considered of 25 genotypes / varieties collected from different region of states of India and maintained at the Division of Vegetable Science. College of Horticulture and Forestry, Jhalarapatan City, Jhalawar, Rajasthan, India.

The experiment was laid out in a Randomized Block Design (RBD) with three replications. Each genotype was sown in raised bed of $1 \times 1$ $\mathrm{m}^{2}$ area on June, 2017 to March, 2018. A distance of $45 \mathrm{~cm}$ between rows and $20 \mathrm{~cm}$ between plants was maintained. All the cultural operations like field preparation, fertigation, irrigation, weeding, plant protection measures etc. were carried out as per recommendations in order to raise a successful crop.
The observation was recorded at maximum growth stage and after harvesting on randomly selected 5 plants in each replication for all the characters viz., plant height $(\mathrm{cm})$, number of tillers per plant, number of leaves per plant, leaf width $(\mathrm{cm})$, leaf length $(\mathrm{cm})$, girth of stem $(\mathrm{cm})$, girth of mother rhizomes per plant $(\mathrm{cm})$, girth of primary rhizomes per plant $(\mathrm{cm})$, girth of secondary rhizomes per plant $(\mathrm{cm})$, length of mother rhizomes per plant $(\mathrm{cm})$, length of primary rhizomes per plant $(\mathrm{cm})$, length of secondary rhizomes per plant $(\mathrm{cm})$, number of mother rhizomes per plant, number of primary rhizomes per plant, number of secondary rhizomes per plant, fresh weight of primary rhizomes per plant $(\mathrm{g})$, fresh weight of secondary rhizomes per plant (g), dry weight of rhizomes per plant (g), days to harvest, curcumin content (\%) and yield per plant $(\mathrm{g})$. The curcumin content was estimated by adopting the spectrometer method given by Geethanjali et al., (2016). The data statistically analyzed for variance using the standard procedure by Gomez and Gomez (1983). The genotypic and phenotypic coefficient of variations was analyzed as suggested by Burton and De-Vane (1953). Heritability in broad sense and expected genetic advance as percentage of mean was calculated using the method suggested by Johnson et al., (1955).

\section{Results and Discussion}

\section{Mean performance and genetic variability}

In the present investigation, significant differences among the genotypes were obtained for all the characters, suggesting thereby the presence of significant variation among the genotypes of these traits. Based on the mean value with respect to characters, the genotype JWRH-5 was the higher yielder followed by JWRH-8 and JWRH-7 (Table 14). 
Table.1 Mean performance of turmeric genotypes for different plant growth characters

\begin{tabular}{|c|c|c|c|c|c|c|}
\hline Genotypes & $\begin{array}{l}\text { Plant height } \\
(\mathrm{cm})\end{array}$ & $\begin{array}{c}\text { Number of } \\
\text { tillers per } \\
\text { plant }\end{array}$ & $\begin{array}{c}\text { Number of } \\
\text { leaves per } \\
\text { plant }\end{array}$ & $\begin{array}{l}\text { Leaf width } \\
\text { (cm) }\end{array}$ & $\begin{array}{l}\text { Leaf length } \\
(\mathrm{cm})\end{array}$ & $\begin{array}{c}\text { Girth of } \\
\text { stem }(\mathrm{cm})\end{array}$ \\
\hline Krishna & 137.69 & 3.00 & 20.22 & 14.66 & 75.86 & 5.31 \\
\hline JWRH-1 & 143.80 & 4.83 & 17.31 & 15.25 & 84.16 & 5.95 \\
\hline Laka Dong & 146.71 & 4.73 & 21.48 & 17.26 & 74.30 & 6.75 \\
\hline JWRH-2 & 144.13 & 4.00 & 20.22 & 17.15 & 90.03 & 5.35 \\
\hline JWRH-10 & 145.59 & 2.31 & 19.25 & 16.91 & 83.39 & 6.38 \\
\hline JWRH -11 & 142.16 & 1.06 & 17.78 & 17.98 & 74.40 & 7.08 \\
\hline GNT-1 & 150.16 & 3.80 & 22.90 & 18.94 & 82.27 & 6.07 \\
\hline JWRH-3 & 139.86 & 3.52 & 20.33 & 16.69 & 82.87 & 5.40 \\
\hline JWRH-4 & 142.78 & 3.84 & 19.67 & 15.45 & 83.82 & 5.88 \\
\hline JWRH-7 & 161.05 & 3.88 & 19.96 & 16.60 & 93.73 & 6.07 \\
\hline JWRH-9 & 153.26 & 3.35 & 20.70 & 20.05 & 92.30 & 6.20 \\
\hline JWRH-6 & 148.05 & 4.43 & 21.18 & 18.93 & 80.18 & 5.93 \\
\hline Patangadi & 142.90 & 3.84 & 20.18 & 18.36 & 79.56 & 7.01 \\
\hline Erode local & 147.00 & 3.46 & 21.63 & 19.84 & 90.14 & 6.85 \\
\hline Salem & 120.20 & 1.51 & 21.22 & 15.26 & 70.80 & 5.19 \\
\hline Belogaum local & 125.36 & 2.55 & 21.74 & 15.28 & 76.58 & 5.80 \\
\hline JWRH-13 & 140.75 & 3.61 & 22.85 & 17.25 & 73.17 & 4.87 \\
\hline JWRH-12 & 136.43 & 2.94 & 25.30 & 16.71 & 79.14 & 6.61 \\
\hline Keshar & 148.16 & 4.90 & 21.14 & 18.32 & 80.37 & 6.94 \\
\hline JWRH-17 & 156.68 & 3.66 & 23.47 & 18.31 & 84.38 & 7.36 \\
\hline JWRH-5 & 162.83 & 0.66 & 22.48 & 19.39 & 85.44 & 8.22 \\
\hline JWRH-8 & 156.33 & 0.33 & 21.78 & 20.22 & 80.53 & 8.86 \\
\hline JWRH-14 & 147.53 & 3.08 & 20.70 & 21.69 & 73.98 & 6.17 \\
\hline JWRH-15 & 145.66 & 3.06 & 21.41 & 15.52 & 73.48 & 4.83 \\
\hline JWRH-16 & 132.36 & 3.83 & 21.10 & 17.39 & 71.47 & 4.81 \\
\hline Mean & 144.70 & 3.21 & 21.04 & 17.57 & 80.65 & 6.23 \\
\hline Range & $\begin{array}{c}120.20- \\
162.83\end{array}$ & $0.33-4.90$ & $17.31-25.30$ & $\begin{array}{l}14.66- \\
21.69\end{array}$ & $70.80-93.73$ & $4.81-8.86$ \\
\hline SE $( \pm)$ & 2.28 & 0.30 & 0.66 & 0.41 & 0.59 & 0.29 \\
\hline CD (5\%) & 6.51 & 0.86 & 1.90 & 1.18 & 1.70 & 0.84 \\
\hline $\mathrm{CV}$ & 2.74 & 16.45 & 5.50 & 4.12 & 1.28 & 8.23 \\
\hline
\end{tabular}


Int.J.Curr.Microbiol.App.Sci (2018) 7(7): 3169-3176

Table.2 Mean performance of turmeric genotypes for characters of mother and primary rhizomes

\begin{tabular}{|c|c|c|c|c|c|c|c|}
\hline & \multicolumn{3}{|c|}{ Mother rhizomes } & \multicolumn{3}{c|}{ Primary rhizomes } & \\
\hline Genotypes & $\begin{array}{c}\text { Number } \\
\text { per plant }\end{array}$ & $\begin{array}{c}\text { Girth } \\
(\mathrm{cm})\end{array}$ & $\begin{array}{c}\text { Length } \\
(\mathrm{cm})\end{array}$ & $\begin{array}{c}\text { Number } \\
\text { per plant }\end{array}$ & $\begin{array}{c}\text { Girth } \\
(\mathrm{cm})\end{array}$ & $\begin{array}{c}\text { Length } \\
(\mathrm{cm})\end{array}$ & $\begin{array}{c}\text { Fresh weight } \\
\text { per plant }\end{array}$ \\
\hline Krishna & 0.96 & 2.74 & 5.06 & 6.33 & 2.74 & 6.68 & 250.49 \\
\hline JWRH-1 & 1.07 & 2.04 & 3.51 & 6.96 & 2.04 & 6.94 & 128.85 \\
\hline Laka Dong & 1.01 & 2.00 & 5.47 & 7.33 & 2.00 & 6.43 & 240.26 \\
\hline JWRH-2 & 1.05 & 2.72 & 6.46 & 7.93 & 1.86 & 7.66 & 210.26 \\
\hline JWRH-10 & 1.12 & 2.45 & 4.99 & 6.76 & 2.29 & 8.29 & 175.74 \\
\hline JWRH -11 & 1.01 & 4.70 & 12.04 & 9.80 & 2.44 & 7.01 & 180.22 \\
\hline GNT-1 & 1.23 & 3.37 & 3.81 & 9.43 & 2.20 & 6.69 & 226.66 \\
\hline JWRH-3 & 1.10 & 1.70 & 6.12 & 10.82 & 1.73 & 7.96 & 158.63 \\
\hline JWRH-4 & 1.05 & 2.61 & 4.57 & 6.73 & 2.61 & 9.14 & 243.48 \\
\hline JWRH-7 & 1.04 & 2.98 & 4.59 & 5.83 & 2.98 & 6.82 & 287.06 \\
\hline JWRH-9 & 1.06 & 2.12 & 4.77 & 10.56 & 2.09 & 6.78 & 178.51 \\
\hline JWRH-6 & 1.10 & 2.05 & 4.81 & 6.46 & 2.40 & 6.71 & 337.43 \\
\hline Patangadi & 1.16 & 2.20 & 5.49 & 7.52 & 2.20 & 7.08 & 203.33 \\
\hline Erode local & 1.03 & 3.41 & 5.76 & 10.63 & 2.10 & 8.20 & 248.85 \\
\hline Salem & 1.21 & 2.60 & 3.99 & 4.96 & 2.60 & 5.99 & 128.59 \\
\hline Belogaum & 1.21 & 2.83 & 4.18 & 7.82 & 2.61 & 6.01 & 102.83 \\
\hline local & & & & & & & \\
\hline JWRH-13 & 1.10 & 3.80 & 6.36 & 5.98 & 2.23 & 8.47 & 288.33 \\
\hline JWRH-12 & 1.39 & 2.78 & 6.16 & 5.73 & 2.56 & 7.35 & 121.96 \\
\hline Keshar & 1.09 & 1.93 & 5.53 & 6.20 & 2.45 & 8.34 & 210.11 \\
\hline JWRH-17 & 1.10 & 2.14 & 5.33 & 10.86 & 2.07 & 6.80 & 203.70 \\
\hline JWRH-5 & 1.14 & 3.81 & 10.06 & 9.96 & 2.70 & 7.96 & 265.95 \\
\hline JWRH-8 & 1.00 & 3.74 & 10.37 & 11.59 & 2.77 & 7.23 & 221.78 \\
\hline JWRH-14 & 1.02 & 2.64 & 5.37 & 5.87 & 2.64 & 8.60 & 210.48 \\
\hline JWRH-15 & 1.40 & 3.40 & 6.50 & 5.74 & 2.41 & 7.84 & 140.15 \\
\hline JWRH-16 & 1.30 & 1.68 & 5.55 & 6.28 & 2.01 & 6.88 & 230.38 \\
\hline Mean & 1.12 & 2.74 & 5.87 & 7.76 & 2.35 & 7.35 & 207.76 \\
\hline Range & $0.96-1.40$ & $1.68-$ & $3.51-$ & $4.96-11.59$ & $1.73-$ & $5.99-$ & $102.83-$ \\
\hline CDE (5) & 0.03 & 0.23 & 0.38 & 0.35 & 0.19 & 0.34 & 8.64 \\
\hline CV & 0.08 & 0.65 & 1.09 & 1.01 & 0.56 & 0.97 & 24.58 \\
\hline & 4.64 & 14.61 & 11.29 & 7.92 & 14.61 & 8.10 & 7.20 \\
\hline & & & & & & \\
\hline
\end{tabular}


Table.3 Mean performance of turmeric genotypes for characters of secondary rhizomes and days to harvest

\begin{tabular}{|c|c|c|c|c|c|}
\hline \multirow[t]{2}{*}{ Genotypes } & \multicolumn{4}{|c|}{ Secondary rhizomes } & \multirow[b]{2}{*}{ Days to harvest } \\
\hline & $\begin{array}{l}\text { Number } \\
\text { per plant }\end{array}$ & $\begin{array}{l}\text { Girth } \\
(\mathrm{cm})\end{array}$ & $\begin{array}{l}\text { Length } \\
(\mathrm{cm})\end{array}$ & $\begin{array}{l}\text { Fresh weight per } \\
\text { plant (g) }\end{array}$ & \\
\hline Krishna & 10.51 & 2.36 & 5.19 & 122.48 & 251.52 \\
\hline JWRH-1 & 12.81 & 2.52 & 6.60 & 190.11 & 247.33 \\
\hline Laka Dong & 16.73 & 1.98 & 5.84 & 136.99 & 245.74 \\
\hline JWRH-2 & 9.72 & 1.63 & 5.41 & 100.41 & 264.79 \\
\hline JWRH-10 & 12.96 & 1.88 & 5.43 & 309.33 & 253.74 \\
\hline JWRH -11 & 14.36 & 1.67 & 4.17 & 105.26 & 259.66 \\
\hline GNT-1 & 14.33 & 1.60 & 6.10 & 145.29 & 221.47 \\
\hline JWRH-3 & 16.14 & 1.75 & 6.66 & 162.16 & 266.58 \\
\hline JWRH-4 & 12.16 & 2.66 & 7.55 & 253.52 & 250.33 \\
\hline JWRH-7 & 10.48 & 2.65 & 5.60 & 220.37 & 250.7 \\
\hline JWRH-9 & 15.80 & 2.04 & 4.75 & 103.62 & 261.33 \\
\hline JWRH-6 & 14.71 & 2.40 & 6.08 & 148.59 & 257.45 \\
\hline Patangadi & 17.04 & 2.15 & 7.60 & 213.66 & 228.66 \\
\hline Erode local & 12.81 & 1.77 & 5.36 & 168.44 & 218 \\
\hline Salem & 6.02 & 1.85 & 2.75 & 98.44 & 234.21 \\
\hline Belogaum local & 8.81 & 2.49 & 6.81 & 106.85 & 248.78 \\
\hline JWRH-13 & 12.02 & 2.74 & 6.83 & 155.11 & 246.66 \\
\hline JWRH-12 & 9.97 & 2.42 & 6.09 & 286.03 & 229.82 \\
\hline Keshar & 10.06 & 2.40 & 6.43 & 121.40 & 233.04 \\
\hline JWRH-17 & 16.31 & 1.85 & 4.46 & 105.67 & 240.74 \\
\hline JWRH-5 & 14.41 & 1.88 & 4.92 & 65.31 & 220.66 \\
\hline JWRH-8 & 21.02 & 1.88 & 5.28 & 110.07 & 247 \\
\hline JWRH-14 & 13.73 & 2.41 & 6.92 & 117.48 & 246.85 \\
\hline JWRH-15 & 11.06 & 2.44 & 5.87 & 225.41 & 267.44 \\
\hline JWRH-16 & 11.44 & 2.09 & 6.57 & 245.22 & 219.77 \\
\hline Mean & 13.01 & 2.14 & 5.81 & 160.69 & 244.49 \\
\hline Range & $6.02-21.02$ & $1.60-2.74$ & $2.75-7.60$ & $65.31-309.33$ & $218.00-267.44$ \\
\hline SE $( \pm)$ & 0.81 & 0.16 & 0.47 & 5.57 & 3.50 \\
\hline CD (5\%) & 2.31 & 0.48 & 1.35 & 16.71 & 9.96 \\
\hline $\mathrm{CV}$ & 10.84 & 13.64 & 14.24 & 6.33 & 2.48 \\
\hline
\end{tabular}


Table.4 Mean performance of turmeric genotypes for yield and different quality characters

\begin{tabular}{|c|c|c|c|}
\hline Genotypes & Yield per plant (g) & $\begin{array}{c}\text { Dry weight of } \\
\text { rhizomes per plant } \\
\text { (g) }\end{array}$ & $\begin{array}{c}\text { Curcumin } \\
\text { content }(\%)\end{array}$ \\
\hline Krishna & 449.16 & 56.43 & 2.02 \\
\hline JWRH-1 & 428.82 & 87.01 & 2.12 \\
\hline Laka Dong & 390.20 & 81.65 & 2.05 \\
\hline JWRH-2 & 445.20 & 93.39 & 1.60 \\
\hline JWRH-10 & 518.63 & 94.14 & 2.14 \\
\hline JWRH -11 & 514.29 & 105.68 & 1.69 \\
\hline GNT-1 & 423.64 & 112.04 & 3.44 \\
\hline JWRH-3 & 375.31 & 24.68 & 2.24 \\
\hline JWRH-4 & 538.92 & 124.49 & 2.16 \\
\hline JWRH-7 & 549.66 & 123.44 & 4.15 \\
\hline JWRH-9 & 281.49 & 70.55 & 1.78 \\
\hline JWRH-6 & 434.16 & 35.54 & 1.66 \\
\hline Patangadi & 475.15 & 100.27 & 2.80 \\
\hline Erode local & 512.61 & 121.66 & 2.65 \\
\hline Salem & 292.48 & 74.36 & 2.64 \\
\hline Belogaum local & 528.33 & 119.33 & 2.83 \\
\hline JWRH-13 & 457.06 & 104.97 & 2.05 \\
\hline JWRH-12 & 422.71 & 101.33 & 2.41 \\
\hline Keshar & 510.78 & 107.48 & 2.60 \\
\hline JWRH-17 & 510.41 & 101.40 & 2.67 \\
\hline JWRH-5 & 825.06 & 200.90 & 2.39 \\
\hline JWRH-8 & 698.00 & 188.46 & 1.93 \\
\hline JWRH-14 & 510.50 & 120.03 & 3.15 \\
\hline JWRH-15 & 465.69 & 114.05 & 2.12 \\
\hline JWRH-16 & 284.08 & 56.40 & 1.74 \\
\hline Mean & 473.69 & 100.79 & 2.36 \\
\hline Range & $281.49-825.06$ & $24.68-200.90$ & $1.60-4.15$ \\
\hline SE $( \pm)$ & 4.16 & 2.41 & 0.05 \\
\hline CD (5\%) & 11.83 & 6.88 & 0.14 \\
\hline $\mathrm{CV}$ & 1.52 & 4.15 & 3.66 \\
\hline
\end{tabular}


Table 1.5 Estimates of phenotypic and genotypic coefficients of variability, heritability, genetic advance and genetic gain for different characters in turmeric

\begin{tabular}{|c|c|c|c|c|c|c|}
\hline \multirow[t]{2}{*}{ S.No. } & \multirow[t]{2}{*}{ Characters } & \multicolumn{2}{|c|}{$\begin{array}{l}\text { Coefficients of } \\
\text { variability }(\%)\end{array}$} & \multirow[t]{2}{*}{$\begin{array}{c}\text { Heritability } \\
(\%)\end{array}$} & \multirow[t]{2}{*}{$\begin{array}{l}\text { Genetic } \\
\text { advance }\end{array}$} & \multirow[t]{2}{*}{$\begin{array}{c}\text { Genetic gain } \\
(\%)\end{array}$} \\
\hline & & GCV & PCV & & & \\
\hline 1 & Plant height (cm) & 6.60 & 7.15 & 85.31 & 18.18 & 12.56 \\
\hline 2 & Number of tillers per plant & 37.01 & 40.50 & 83.50 & 2.23 & 69.67 \\
\hline 3 & Number of leaves per plant & 7.36 & 9.18 & 64.10 & 2.55 & 12.14 \\
\hline 4 & Leaf width (cm) & 10.16 & 10.96 & 85.90 & 3.41 & 19.39 \\
\hline 5 & Leaf length (cm) & 8.02 & 8.13 & 97.50 & 13.17 & 16.33 \\
\hline 6 & Girth of stem (cm) & 15.51 & 17.56 & 78.01 & 1.76 & 28.22 \\
\hline 7 & $\begin{array}{l}\text { Girth of mother rhizomes } \\
\text { per plant }(\mathrm{cm})\end{array}$ & 26.57 & 30.33 & 76.82 & 1.31 & 47.97 \\
\hline 8 & $\begin{array}{l}\text { Girth of primary rhizomes } \\
\text { per plant }(\mathrm{cm})\end{array}$ & 10.57 & 18.03 & 34.34 & 0.30 & 12.76 \\
\hline 9 & $\begin{array}{l}\text { Girth of secondary } \\
\text { rhizomes per plant (cm) }\end{array}$ & 14.62 & 20.00 & 53.51 & 0.47 & 22.03 \\
\hline 10 & $\begin{array}{l}\text { Length of mother rhizomes } \\
\text { per plant }(\mathrm{cm})\end{array}$ & 34.34 & 36.15 & 90.24 & 3.95 & 67.20 \\
\hline 11 & $\begin{array}{l}\text { Length of primary } \\
\text { rhizomes per plant (cm) }\end{array}$ & 10.42 & 13.20 & 62.30 & 1.24 & 16.96 \\
\hline 12 & $\begin{array}{l}\text { Length of secondary } \\
\text { rhizomes per plant }(\mathrm{cm})\end{array}$ & 17.06 & 22.22 & 58.94 & 1.56 & 26.98 \\
\hline 13 & $\begin{array}{l}\text { Number of mother } \\
\text { rhizomes per plant }\end{array}$ & 9.96 & 10.99 & 82.20 & 0.20 & 18.61 \\
\hline 14 & $\begin{array}{l}\text { Number of primary } \\
\text { rhizomes per plant }\end{array}$ & 25.79 & 26.98 & 91.47 & 3.94 & 50.79 \\
\hline 15 & $\begin{array}{l}\text { Number of secondary } \\
\text { rhizomes per plant }\end{array}$ & 23.85 & 26.20 & 82.91 & 5.82 & 44.74 \\
\hline 16 & $\begin{array}{l}\text { Fresh weight of primary } \\
\text { rhizomes per plant (g) }\end{array}$ & 27.55 & 28.47 & 93.60 & 114.08 & 54.91 \\
\hline 17 & $\begin{array}{l}\text { Fresh weight of secondary } \\
\text { rhizomes per plant (g) }\end{array}$ & 40.46 & 40.96 & 97.68 & 132.34 & 82.36 \\
\hline 18 & $\begin{array}{l}\text { Dry weight of rhizomes per } \\
\text { plant (g) }\end{array}$ & 38.65 & 38.88 & 98.96 & 79.80 & 79.18 \\
\hline 19 & Days to harvest & 6.01 & 6.50 & 85.44 & 28.00 & 11.45 \\
\hline 20 & Curcumin content $(\%)$ & 25.33 & 25.60 & 98.00 & 1.22 & 51.66 \\
\hline 21 & Yield per plant (g) & 24.69 & 24.74 & 99.62 & 240.53 & 50.77 \\
\hline
\end{tabular}


Int.J.Curr.Microbiol.App.Sci (2018) 7(7): 3169-3176 
Among all the characters, high phenotypic and genotypic coefficient of variation was recorded for per cent fresh weight of secondary rhizomes per plant followed by dry weight of rhizomes per plant, number of tillers per plant and length of mother rhizomes per plant. These results are in conformity with the results of pervious work Singh et al., (2003). However, the low estimates of PCV observed for characters like length of primary rhizomes per plant, number of mother rhizomes per plant, leaf length and width, number of leaves per plant, plant height and days to harvest indicated that the genotypes used had less genetic variability for these characters. While, girth of primary and secondary rhizomes per plant, length of primary rhizomes per plant, leaf width and leaf length, number of mother rhizomes per plant, number of leaves per plant, plant height and days to harvest, GCV were low.

\section{References}

Burton, G.W. and De-Vane, E.W. (1953). Estimating heritability in tall fescue (Festuca arundiancea) from replicated clonal material. Proejtunniens, 9(22): 12-15.

Gomez, K.A. and Gomez, A.A. (1983).
Statistical Procedures Agric. Res. John Wiley and Sons Inc., New York. pp. 357-427.

Johanson, H.W., Robinson, H.F. and Comstock, R.E. (1955). Estimates of genetic and environmental variability in soybean. Agron J., 47: 314-318.

Prajapati, K.N., Patel, M.A., Patel, J.R., Joshi, N.R., Patel, A.D. and Patel, J.R. (2014). Genetic variability, character association and path coefficient analysis in turmeric (Curcuma longa L.). Electronic J. Plant Breed., 5(1): 131-137.

Sato, D. (1960). The karyotype analysis in zingiberales with special reference to prokaryotype and stable karyotype. Scientific paper of the college of General education, Univ. Tokyo., 10(2): 225-243.

Singh, Y., Mittal, P. and Katoch V. (2003). Genetic variability and heritability in turmeric (Curcuma longa L.). Himachal J. Agric. Res., 29(1\&2): 3134.

Geethanjali, A., Lalitha, P. and Jannathul, F.M. (2016). Analysis of Curcumin Content of Turmeric Samples from Various States of India. Int. J. Pharma Chemi. Res., 2(1): 55-62.

\section{How to cite this article:}

Poonam, I. B. Maurya, Manoj Sharma, A. Kavita, Bhim Singh, Bhuri Singh, Priyanka Kumawat and Anita Verma. 2018. Studies on Genetic Variability, Hertability and Genetic Advance in Turmeric (Curcuma longa L.) Int.J.Curr.Microbiol.App.Sci. 7(07): 3169-3176. doi: https://doi.org/10.20546/ijcmas.2018.707.370 